PROCEEDINGS OF THE

AMERICAN MATHEMATICAL SOCIETY

Volume 129, Number 5, Pages 1535-1544

S 0002-9939(00)05664-1

Article electronically published on October 10, 2000

\title{
PRODUCTS OF MICHAEL SPACES AND COMPLETELY METRIZABLE SPACES
}

\author{
DENNIS K. BURKE AND ROMAN POL
}

(Communicated by Alan Dow)

\begin{abstract}
For disjoint subsets $A, C$ of $[0,1]$ the Michael space $M(A, C)=$ $A \cup C$ has the topology obtained by isolating the points in $C$ and letting the points in $A$ retain the neighborhoods inherited from $[0,1]$. We study normality of the product of Michael spaces with complete metric spaces. There is a ZFC example of a Lindelöf Michael space $M(A, C)$, of minimal weight $\aleph_{1}$, with $M(A, C) \times B\left(\aleph_{0}\right)$ Lindelöf but with $M(A, C) \times B\left(\aleph_{1}\right)$ not normal. $\left(B\left(\aleph_{\alpha}\right)\right.$ denotes the countable product of a discrete space of cardinality $\aleph_{\alpha}$.) If $M(A)$ denotes $M(A,[0,1] \backslash A)$, the normality of $M(A) \times B\left(\aleph_{0}\right)$ implies the normality of $M(A) \times S$ for any complete metric space $S$ (of arbitrary weight). However, the statement " $M(A, C) \times B\left(\aleph_{1}\right)$ normal implies $M(A, C) \times B\left(\aleph_{2}\right)$ normal" is axiom sensitive.
\end{abstract}

\section{INTRODUCTION}

The Michael space $M(A, C)$, associated with a pair of disjoint sets $A, C$ in the unit interval $I=[0,1]$, is the set $A \cup C$ equipped with the topology consisting of all sets $(U \cap(A \cup C)) \cup C^{\prime}$ where $U$ is open in $I$ and $C^{\prime} \subseteq C$. That is, isolate the points in $C$ and let the points in $A$ retain the usual neighborhoods from the topology inherited from $I$. We shall write $M(A)=M(A, I \backslash A)$. The usual Michael Line would be homeomorphic to the space $M(\mathbb{Q} \cap(0,1))$, where $\mathbb{Q}$ is the set of rational numbers. E. Michael introduced this example in [M1] as a paracompact space whose product with the separable complete metric space of irrational numbers $\mathbb{P}$ is not normal. Using CH, Michael ([M1], M2]) also introduced a Lindelöf subspace $X$ of the Michael Line having the property that $X \times \mathbb{P}$ is not normal. This prompted him to ask the question, still unanswered today, of whether there exists a ZFC example of a Lindelöf space whose product with $\mathbb{P}$ is not normal. While our results do not address this question directly, it is related to the purpose of the example given in Section 2, where a ZFC example is given of a Lindelöf Michael space $M(A, C)$, of weight $\aleph_{1}$, with $M(A, C) \times B\left(\aleph_{0}\right)$ Lindelöf but with $M(A, C) \times B\left(\aleph_{1}\right)$ not normal. (Notation for the Baire Space $B\left(\aleph_{1}\right)$ is explained in a later paragraph of the Introduction. The issue here is that it is a complete metric space of weight $\aleph_{1}$.)

Received by the editors March 8, 1998 and, in revised form, July 28, 1999.

1991 Mathematics Subject Classification. Primary 54E50, 54E52, 54D15.

Key words and phrases. Michael space, product spaces, normal, completely metrizable, Baire space, absolute $G_{\delta}$.

The results in this note were obtained while the second author was a Visiting Professor at Miami University. The author would like to express his gratitude to the Department of Mathematics and Statistics of Miami University for their hospitality. 
The first ZFC example of a Lindelöf space and a completely metrizable space having a product which is nonnormal and of minimal weight $\aleph_{1}$ was given by Lawrence [L2]. The example in Section 2, with relatively simple structure, gives a much different approach to the construction. As pointed out by Lawrence, the significance of the minimal weight $\aleph_{1}$ is that it is not possible to construct a ZFC example of a Lindelöf space, of weight $\aleph_{1}$, having a nonnormal product with $\mathbb{P}([$ L1] $)$.

In Section 3 we show that for Michael spaces of type $M(A)$, the property of normality of $M(A) \times S$, for $S$ a complete metric space (of arbitrary weight), is actually implied by the normality of $M(A) \times \mathbb{P}$. This is in contrast with the behavior of the main example $M(A, C)$ given in Section 2. In Section 4 we find that the expectation of normality of $M(A, C) \times B\left(\aleph_{1}\right)$ implying the normality of $M(A, C) \times$ $B\left(\aleph_{2}\right)$ is axiom sensitive. That is, it is consistent that there is a Michael space $M(A, C)$ such that $M(A, C) \times B\left(\aleph_{1}\right)$ is normal but $M(A, C) \times B\left(\aleph_{2}\right)$ is not normal. It is also consistent (assuming a supercompact cardinal) that $M(A, C) \times B\left(\aleph_{1}\right)$ normal implies that $M(A, C) \times S$ is normal for every complete metric space $S$.

By the Baire Space $B\left(\aleph_{\alpha}\right)$ we mean the countable product $B\left(\aleph_{\alpha}\right)=D^{\mathbb{N}}$ where $D$ is the discrete space of cardinality $\aleph_{\alpha}$ and $\mathbb{N}$ is the set of positive integers. Recall that the space $B\left(\aleph_{0}\right)=\mathbb{N}^{\mathbb{N}}$ is homeomorphic to the space $\mathbb{P}$ of irrational numbers. Any completely metrizable space $S$ of weight $\aleph_{\alpha}$ is a closed image of $B\left(\aleph_{\alpha}\right)$. It follows that, for every $X$, the normality of $X \times B\left(\aleph_{\alpha}\right)$ implies the normality of $X \times S$.

Any other topological or set theoretic notation used but not defined in this note can be found in $[\mathrm{E}]$ or $[\mathrm{Ku}]$.

$$
\begin{gathered}
\text { 2. A MichaEL SPACE } M(A, C) \text { with } \\
M(A, C) \times B\left(\aleph_{0}\right) \text { LindelöF BUt } M(A, C) \times B\left(\aleph_{1}\right) \text { NOt NORMal }
\end{gathered}
$$

We begin with the following useful observation concerning Michael spaces.

Remark 2.1. The product $M(A, C) \times S$ of a Michael space and a metrizable space $S$ is normal if and only if for each $F \subseteq C \times S$, closed in $M(A, C) \times S$, the pair $F$ and $A \times S$ can be separated in $M(A, C) \times S$ by disjoint open sets.

Proof. Indeed, to see the nontrivial direction of this remark, let $E, F$ be disjoint closed subsets of $M(A, C) \times S$. If $E^{\prime}=E \cap(A \times S)$ and $F^{\prime}=F \cap(A \times S)$, then $E^{\prime}, F^{\prime}$ are separated with respect to the completely normal metric topology on $(A \cup C) \times S$, so there exist disjoint metric open sets $U, V$, in $(A \cup C) \times S$, containing $E^{\prime}, F^{\prime}$, respectively. Now, $T=(E \backslash U) \cup(F \backslash V)$ is closed in $M(A, C) \times S$ and we are assuming that there exist open $W$ such that $T \subseteq W$ and $\bar{W} \cap(A \times S)=\emptyset$. Then $U^{\prime}=U \backslash \bar{W}, V^{\prime}=V \backslash \bar{W}$ are disjoint open sets in $M(A, C) \times S$ with $E^{\prime} \subseteq U^{\prime}, F^{\prime} \subseteq V^{\prime}$ and

$$
\overline{U^{\prime}} \cap(F \backslash A \times S)=\emptyset, \overline{V^{\prime}} \cap(E \backslash A \times S)=\emptyset .
$$

Continue by covering the "vertical sections" of $E \cap(C \times S)$ and $F \cap(C \times S)$ in $C \times S$. For this, use normality of $S$ to find, for every $z \in C$, disjoint open sets $G_{z}, H_{z}$ in $S$ such that

$$
E \cap(\{z\} \times S) \subseteq\{z\} \times G_{z} \subseteq\{z\} \times S \backslash \overline{V^{\prime}}
$$

and

$$
F \cap(\{z\} \times S) \subseteq\{z\} \times H_{z} \subseteq\{z\} \times S \backslash \overline{U^{\prime}}
$$


Then, $U^{\prime} \cup\left(\bigcup_{z \in C}\{z\} \times G_{z}\right)$ and $V^{\prime} \cup\left(\bigcup_{z \in C}\{z\} \times H_{z}\right)$ are the desired disjoint open sets separating $E, F$ in $M(A, C) \times S$.

To describe a Michael space with the properties in the title let us represent the irrationals $\mathbb{P}$ in the unit interval $I$ as the countable product $\mathbb{P}=L^{\mathbb{N}}$ with $L$ homeomorphic to $\mathbb{P}$, and let $T \subseteq L$ be a set of cardinality $\aleph_{1}$ without any Cantor subset. Let $B\left(\aleph_{1}\right)=D^{\mathbb{N}}$ with $D$ discrete of cardinality $\aleph_{1}$. Now fix a bijection $\phi: D \rightarrow T$ and let

$$
f=\phi \times \phi \times \cdots: D^{\mathbb{N}} \rightarrow T^{\mathbb{N}} .
$$

Following Stone [S] let us choose a non- $\sigma$-discrete set $E=\left\{e_{\alpha}: \alpha<\omega_{1}\right\} \subseteq$ $B\left(\aleph_{1}\right)$, all separable subsets of which are countable. We set

$$
A=\mathbb{P} \backslash T^{\mathbb{N}} \text { and } C=f(E) \subseteq T^{\mathbb{N}} .
$$

Let us check that

$$
M(A, C) \times \mathbb{P} \text { is Lindelöf. }
$$

Since the neighborhoods of the points in $A \times \mathbb{P}$ are Euclidean, and $A \times \mathbb{P}$ is Lindelöf, it is enough to show that if $U$ is open in $\mathbb{P} \times \mathbb{P}$ and $A \times \mathbb{P} \subseteq U$, then $F=\mathbb{P} \times \mathbb{P} \backslash U$ intersects only countably many sections $\{c\} \times \mathbb{P}, c \in C$. Let $H$ be the projection of $F$ onto the first coordinate. Then $H$ is an analytic set in $T^{\mathbb{N}}$ (cf. (2)), and so is the projection $H_{i}$ of $H$ onto the $i$ th coordinate. Since $H_{i}$ contains no Cantor set, it is countable. Therefore, $H \subseteq H_{1} \times H_{2} \times \cdots$, with every $H_{i}$ countable, and by (1), $f^{-1}(H) \subseteq \phi^{-1}\left(H_{1}\right) \times \phi^{-1}\left(H_{2}\right) \times \cdots$ is separable in $B\left(\aleph_{1}\right)$. It follows that $f^{-1}(H) \cap E$ is countable, and so is the set $C^{\prime}=H \cap C$ (cf. (2)). Because $F$ intersects $\{c\} \times \mathbb{P}$ only for $c \in C^{\prime}$, this completes our verification of (3).

We now demonstrate that

$$
M(A, C) \times B\left(\aleph_{1}\right) \text { is not normal. }
$$

The set

$$
F=\{(f(z), z): z \in E\}
$$

is the trace of the graph of $f$ on $(A \cup C) \times B\left(\aleph_{1}\right)$ and by the continuity of $f$ the set $F$ is closed in the product (even with $A \cup C$ carrying the topology inherited from $I)$. Let $U$ be an arbitrary open set in $M(A, C) \times B\left(\aleph_{1}\right)$ containing $F$. To get (4), it is enough to show that

$$
\bar{U} \cap\left(A \times B\left(\aleph_{1}\right)\right) \neq \emptyset .
$$

For each $z \in E$, let $U(z)$ be a neighborhood of $z$ in $B\left(\aleph_{1}\right)$ with $\{f(z)\} \times U(z) \subseteq U$. Since $E$ is not $\sigma$-discrete, we show

$$
\begin{aligned}
& \text { there exists } w \in E \text { for which the set } K=\{z \in E: w \in U(z)\} \\
& \text { is uncountable. }
\end{aligned}
$$

To see that (6) is true, assume otherwise. Then $\{U(z) \cap E: z \in E\}$ is index point-countable. For our contradiction it is enough to argue that this shows $E$ is $\sigma$-discrete. We may assume that each $U(z)$ is actually a $\frac{1}{n}$-ball $B\left(z, \frac{1}{n}\right)$, for some $n \in \mathbb{N}$, with respect to a fixed metric. If $E_{n}=\left\{z \in E: U(z)=B\left(z, \frac{1}{n}\right)\right\}$, then each $\left\{U(z) \cap E_{n}: z \in E_{n}\right\}$ index point-countable implies that every $U(z) \cap E_{n}$, for $z \in E_{n}$, is countable. This says that $\left\{U(z) \cap E_{n}: z \in E_{n}\right\}$ witnesses its own local countability. Now, a standard "chaining argument" (cf. [E], 5.3.A) would imply that each $E_{n}$ is $\sigma$-discrete. That shows (6) is true. To complete the 
proof that $M(A, C) \times B\left(\aleph_{1}\right)$ is not normal we observe that by $(3), M(A, C)$ is Lindelöf, so the uncountable set $f(K)$ has an accumulation point $a \in A$. Hence $(a, w) \in \overline{f(K) \times\{w\}} \subseteq \bar{U}$, witnessing (5).

We conclude this section with a formal statement of the example just constructed.

Example 2.2. There is a Lindelöf space $M(A, C)$, of weight $\aleph_{1}$, such that $M(A, C) \times B\left(\aleph_{0}\right)$ is Lindelöf but $M(A, C) \times B\left(\aleph_{1}\right)$ is not normal.

\section{Normality of $M(A) \times B\left(\aleph_{0}\right)$ IMPLiES NORMALITY OF $M(A) \times S$ FOR ANY COMPLETE $S$}

The reasoning in this section will be based on the following lemma.

Lemma 3.1. Let $f: S \rightarrow E$ be a continuous map from a completely metrizable space $S$ to a separable metrizable space $E$, and let $\mathcal{F}$ be a collection of closed subsets of $E$ such that the image under $f$ of any separable set is covered by countably many elements of $\mathcal{F}$. Then, for some $F \in \mathcal{F}, f^{-1}(F)$ has nonempty interior.

Proof. We justify the assertion in two steps.

Step I. Assume that $S$ has weight $\aleph_{1}$, let $\left\{x_{\alpha}: \alpha<\omega_{1}\right\}$ be dense in $S$, and let

$$
S_{\alpha}=\overline{\left\{x_{\beta}: \beta<\alpha\right\}} \text {. }
$$

By the assumption about $f$, the closed separable set $S_{\alpha}$ is covered by countably many sets $f^{-1}(F), F \in \mathcal{F}$, and by the Baire Category Theorem there exist $\phi(\alpha)<$ $\alpha, \varepsilon(\alpha)>0$ and $F_{\alpha} \in \mathcal{F}$ such that

$$
B\left(x_{\phi(\alpha)}, \varepsilon(\alpha)\right) \cap S_{\alpha} \subseteq f^{-1}\left(F_{\alpha}\right),
$$

where $B(x, \varepsilon)$ is the $\varepsilon$-ball about $x$, with respect to a fixed metric in $S$. Since $\phi$ is regressive, there exists an uncountable set $\Lambda \subseteq \omega_{1}$ and $r>0$ with $\phi(\xi)=\alpha$, $\varepsilon(\xi) \geq r$ for all $\xi \in \Lambda$. Letting $a=x_{\alpha}$, we obtain

$$
H_{\xi}=B(a, r) \cap S_{\xi} \subseteq f^{-1}\left(F_{\xi}\right) \text { for } \xi \in \Lambda \text {. }
$$

Since $\bigcup_{\alpha \in \Lambda} f\left(H_{\alpha}\right)$ is separable, and $H_{\alpha} \subseteq H_{\beta}$, for $\alpha<\beta$, there exists $\xi \in \Lambda$ with $f\left(H_{\xi}\right)$ dense in the union, i.e., by $(2), \bigcup_{\alpha \in \Lambda} f\left(H_{\alpha}\right) \subseteq \overline{f\left(H_{\xi}\right)} \subseteq F_{\xi}$, and hence

$$
\bigcup_{\alpha \in \Lambda} H_{\alpha} \subseteq f^{-1}\left(F_{\xi}\right)
$$

But, by (2),

$$
\bigcup_{\alpha \in \Lambda} H_{\alpha}=B(a, r) \cap \bigcup_{\alpha \in \Lambda} S_{\alpha}=B(a, r),
$$

and therefore, $B(a, r) \subseteq f^{-1}\left(F_{\xi}\right)$. This demonstrates the assertion for spaces $S$ of weight $\aleph_{1}$.

Step II. We will now consider the case of arbitrary complete $S$. Aiming at a contradiction, assume that for every $F \in \mathcal{F}$ the preimage $f^{-1}(F)$ has empty interior. We shall define inductively closed separable sets in $S$,

$$
S_{1} \subseteq S_{2} \subseteq \cdots \subseteq S_{\alpha} \subseteq \cdots, \quad \alpha<\omega_{1},
$$

and countable collections $\mathcal{F}_{\alpha} \subseteq \mathcal{F}$ such that

$$
f\left(S_{\alpha}\right) \subseteq \bigcup \mathcal{F}_{\alpha}, \quad \alpha<\omega_{1},
$$


and

$$
f^{-1}(F) \cap S_{\alpha} \subseteq \overline{S_{\alpha+1} \backslash f^{-1}(F)}, \quad \text { for } F \in \bigcup_{\beta \leq \alpha} \mathcal{F}_{\beta} .
$$

Let us start from $S_{0}=\{\emptyset\}$ and $\mathcal{F}_{0}=\left\{F_{0}\right\}$ with any $F_{0} \in \mathcal{F}$. Given $S_{\alpha}, f\left(S_{\alpha}\right)$ being separable, there exists a countable $\mathcal{F}_{\alpha} \subseteq \mathcal{F}$ satisfying (5). For each $F \in \bigcup_{\beta \leq \alpha} \mathcal{F}_{\beta}$, $f^{-1}(F)$ has empty interior and since $S_{\alpha}$ is separable, there is a countable set $C(F)$ disjoint from $f^{-1}(F)$ such that $f^{-1}(F) \cap S_{\alpha} \subseteq \overline{C(F)}$. Then

$$
S_{\alpha+1}=\longdiv { \bigcup \{ C ( F ) : F \in \bigcup _ { \beta \leq \alpha } \mathcal { F } _ { \beta } \} }
$$

satisfies (6). At each limit stage $\xi$, we set $S_{\xi}=\overline{\bigcup_{\alpha<\xi} S_{\alpha}}$.

Having completed the construction, we let

$$
X=\bigcup_{\alpha<\omega_{1}} S_{\alpha}, \quad \mathcal{F}^{\prime}=\bigcup_{\alpha<\omega_{1}} \mathcal{F}_{\alpha}
$$

Then $X$ is closed in $S$; hence it is a complete space of weight $\aleph_{1}$. Let us consider $f \mid X$, the map $f$ restricted to $X$. By (5), the images of separable subsets of $X$ are covered by countable many elements of $\mathcal{F}^{\prime}$. If $F \in \mathcal{F}_{\beta}$ and $x \in f^{-1}(F) \cap S_{\alpha}$, with $\alpha \geq \beta$, then by (6) $x$ is the limit of a sequence from $X \backslash f^{-1}(F)$. It follows that all sets $(f \mid X)^{-1}(F), F \in \mathcal{F}^{\prime}$, have empty interior in $X$, contradicting the fact we have already established in Step I.

Remark 3.2. The reasoning in Step I also yields the following fact. Let $S$ be a union of an increasing sequence of closed separable sets $S_{1} \subseteq S_{2} \subseteq \cdots \subseteq S_{\xi} \subseteq \cdots, \alpha<\omega_{1}$, with each $P_{\xi}=S_{\xi} \backslash \bigcup_{\alpha<\xi} S_{\alpha}$ dense in $S_{\xi}$ and let $\mathcal{F}_{\xi}$ be a countable subcollection of $\mathcal{F}$ covering $f\left(P_{\xi}\right)$. Then for all but non-stationary many $\xi$, there exists $F \in \mathcal{F}_{\xi}$ with the interior of $f^{-1}(F)$ intersecting $P_{\xi}$.

Theorem 3.3. Let $A \subseteq I$ be such that the product of the Michael space $M(A)$ and the irrationals $\mathbb{P}$ is normal. Then the product $M(A) \times S$ with any completely metrizable space $S$ is normal.

Proof. Let $S$ be a completely metrizable space, and let $F \subseteq I \times S$ be a closed set in the product (where $I$ carries the Euclidean topology) disjoint from $A \times S$.

By Remark 2.1, it is enough to check that $F$ can be separated from $A \times S$ by sets open in the product $M(A) \times S$. Let

$$
\mathcal{E}=\{E \subseteq F: \text { there exists an open } W \supseteq A \text { with } E \cap(W \times S)=\emptyset\} .
$$

We shall check that

$$
\mathcal{E} \text { is an exhaustive cover of } F \text {. }
$$

That is, for each nonempty closed subset $H$ of $F$ there exists $E \in \mathcal{E}$ with $E \cap H$ nonempty and relatively open in $H$ [M3]. To this end, let $f: H \rightarrow I$ be the projection onto the first axis restricted to $H$, and let $\mathcal{F}$ be the collection of compact subsets of $I \backslash A$ (with the Euclidean topology). Let $L$ be an arbitrary closed separable subset of $H$. Then $f(L)$ is an analytic subset of $I \backslash A$ and by the Kechris-Louveau-Woodin dichotomy $([\bar{K}]$, Theorem 21.22), either $f(L)$ is covered by countable many elements of $\mathcal{F}$, or else there exists a Cantor set $K \subseteq f(L) \cup A$ such that $K \cap A$ is countable and dense in $K$. But then, by the classical theorem 
of Michael, the closed subspace $K \times B\left(\aleph_{0}\right)$ of $M(A) \times B\left(\aleph_{0}\right)$ is not normal, contradicting the assumption about $M(A)$. It follows that Lemma 3.1 can be applied to $f$ and $\mathcal{F}$ which provides us with $T \in \mathcal{F}$ such that $f^{-1}(T) \cap H$ has a relatively nonempty interior in $H$. Denote this interior by $E$. Then $\overline{f(E)} \cap A=\emptyset$; that is, $E \in \mathcal{E}$.

Having established (9), we can now proceed as follows. By (9), there exist pairwise disjoint elements $E_{\alpha} \in \mathcal{E}, \alpha<\lambda$, such that $F=\bigcup_{\alpha<\lambda} E_{\alpha}$ and, for $\beta<\lambda$, $E_{\beta}$ is open relative to $F \backslash \bigcup_{\alpha<\beta} E_{\alpha}$. Now using the fact that $I$ has a countable base $\mathcal{B}$ and $S$ has a $\sigma$-discrete base $\bigcup_{n=1}^{\infty} \mathcal{G}_{n}$, we see there are closed discrete collections $\mathcal{F}_{n} \subseteq \mathcal{E}, n \in \mathbb{N}$, with $\bigcup_{n \in \mathbb{N}} \mathcal{F}_{n}$ covering $F$ (let us recall that we consider $F$ as a subspace of $I \times S)$. Indeed, for $W \in \mathcal{B}, \beta<\lambda$, and $k \in \mathbb{N}$ let

$$
Z(W, \beta, k)=\bigcup\left\{(W \times G) \cap E_{\beta}: G \in \mathcal{G}_{k} \text { and } F \cap(W \times G) \backslash \bigcup_{\alpha<\beta} E_{\alpha} \subseteq E_{\beta}\right\} .
$$

The collection $\{\overline{Z(W, \beta, k)}: \beta<\lambda\}$ is discrete. Using this type of construction, we can assume in addition that the projection from $I \times S$ onto $S$ takes distinct elements of $\mathcal{F}_{n}$ to disjoint sets and the projection of $\mathcal{F}_{n}$ is a collection discrete in $S$. Since the projection of any $E \in \mathcal{F}_{n}$ onto the first axis can be separated in $M(A)$ from $A$ by open sets (cf. (8)), one readily defines, for $n \in \mathbb{N}$, open sets $W_{n}$ in $M(A) \times S$ with $\bigcup \mathcal{F}_{n} \subseteq W_{n}$ and $\overline{W_{n}} \cap(A \times S)=\emptyset$. Also, since $F$ is closed in $I \times S$, the metric subset $A \times S$ can be covered by countably many open sets $V_{n}$ with $\overline{V_{n}} \cap F=\emptyset$. Then, a pair of disjoint open sets in $M(A) \times S$ separating $F$ and $A \times S$ can be easily defined in a standard way (cf. [E], Lemma 1.5.15).

It may be useful to illustrate Theorem 3.3 with a couple of examples. Michael M2 was aware that if $A \subseteq I$ such that $I \backslash A$ is an uncountable set containing no Cantor sets (for example, if $A$ was a Bernstein subset of $I$ ), then $M(A) \times P$ is normal (in fact, Lindelöf) even though $M(A) \times(I \backslash A)$ is not normal. Hence, for such $A, M(A) \times S$ is normal for all complete metric spaces $S$.

For a different example, suppose $A \subseteq I$ where, for every countable set $D \subseteq A$, there is a $G_{\delta}$-set $H$ with $D \subseteq H \subseteq A$. An example of such $A$ would be the union of an increasing $\omega_{1}$-sequence of $G_{\delta}$-sets as described in [Kur] (page 517). To see that $M(A) \times \mathbb{P}$ is normal look at arbitrary closed $F \subseteq(I \backslash A) \times \mathbb{P}$ (and show $F$ can be separated from $A \times \mathbb{P}$ ). For this set $A$, a Cantor set $K \subseteq I$ cannot have a countable dense subset $D$ with $K \cap A=D$, so the Kechris-Louveau-Woodin Theorem used in the proof of Theorem 3.3 would say that the projection $p_{1}(F)$ into $I$ can be covered by countably many compact sets disjoint from $A$. Say $p_{1}(F) \subseteq \bigcup_{n=1}^{\infty} C_{n}$ where every $C_{n}$ is compact and $C_{n} \cap A=\emptyset$. Now, $\left\{p_{1}^{-1}\left(C_{n}\right): n \in \mathbb{N}\right\}$ is a cover of $F$ consisting of sets which are open and closed in $M(A) \times \mathbb{P}$ and disjoint from $A \times \mathbb{P}$. Also, since we may assume $F$ is closed relative to $I \times \mathbb{P}$, the metric subset $A \times \mathbb{P}$ can be covered by countably many open sets $V_{n}$ with $\overline{V_{n}} \cap F=\emptyset$. Then, a pair of disjoint open sets in $M(A) \times \mathbb{P}$ separating $F$ and $A \times \mathbb{P}$ can be defined in a standard way. Now that $M(A) \times \mathbb{P}$ is seen to be normal, Theorem 3.3 says that $M(A) \times S$ is normal for every complete metric space $S$. The reader may observe here that the above proof that $M(A) \times \mathbb{P}$ is normal is actually a simplified version of the proof of Theorem 3.3 itself.

Although stated in terms of normality, Theorem 3.3 actually gives a result about paracompactness of products $M(A) \times S$, for $S$ completely metrizable, using $M(A) \times$ $\mathbb{P}$ as a test space. Rudin and Starbird $[\overline{\mathrm{RS}}]$ have shown that if $X$ is metrizable and 
$Y$ is paracompact, then $X \times Y$ is normal if and only if $X \times Y$ is paracompact. Since any Michael space $M(A, C)$ is clearly paracompact, the proof of the following corollary is immediate.

Corollary 3.4. For $A \subseteq I, M(A) \times \mathbb{P}$ is normal if and only if $M(A) \times S$ is paracompact for every completely metrizable space $S$.

$$
\begin{aligned}
& \text { 4. The STATEMENT " } M(A, C) \times B\left(\aleph_{1}\right) \text { NORMAL } \\
& \text { IMPLiEs } M(A, C) \times B\left(\aleph_{2}\right) \text { NORMAL" IS AXIOM SENSITIVE }
\end{aligned}
$$

The aim of this section is to substantiate the assertion in the title. We start from the following observation (the notion of an exhaustive collection was recalled in Section 3, following (9)). Recall [E] that a space $E$ is said to be an absolute $G_{\delta}$ if $E$ is a $G_{\delta}$-set in every metric space in which it is embedded.

Lemma 4.1. Let $\mathcal{E}$ be a disjoint decomposition of a metrizable space $X$ such that each countable union of elements of $\mathcal{E}$ is an absolute $G_{\delta}$-set. Then the collection $\mathcal{E}$ is exhaustive, and, in particular, $X$ is completely metrizable.

Proof. Let $H$ be a closed subset of $X$ and let $E \in \mathcal{E}$. Then $E \cap H$ is an $F_{\sigma}$-set in $X$. Otherwise, $E \cap H$ being an absolute $G_{\delta}$-set, a theorem of Hurewicz [H] would yield a Cantor set $K$ in $H$ with $K \backslash E$ countable and dense in $K$. However, $K \backslash E$ is the trace on $K$ of the union of the countable collection $\{G \in \mathcal{E}: G \cap(K \backslash E) \neq \emptyset\}$, i.e., a $G_{\delta}$-set in $K$, which contradicts the Baire Category Theorem.

Arriving at a contradiction, suppose that for each $E \in \mathcal{E}, E \cap H$ has relatively empty interior in $H$ and so $E \cap H$ has a boundary point in $H$. Then one can choose inductively, for $i \in \mathbb{N}$, disjoint sets $E_{i} \in \mathcal{E}$ and points $a_{i} \in E_{i} \cap H$ such that the set $Q=\left\{a_{1}, a_{2}, \cdots\right\}$ has no isolated points (cf. [Ko, proof of Theorem 2). We obtain in this way a completely metrizable space $G=\bar{Q} \cap \bigcup_{i=1}^{\infty} E_{i}$ which can be split into two disjoint dense $G_{\delta}$-sets. This, however, is impossible, by the Baire Theorem.

Now that $\mathcal{E}$ is known to be exhaustive, the fact that $X$ must be completely metrizable follows from Theorem 1.3 in [M3]. This is true because, following terminology from [M3], the (constant) sequence $(\mathcal{E})_{n \in \mathbb{N}}$ is a complete sequence of exhaustive covers.

To show that in some models of set theory the implication is not true we use the following lemma. (Recall $[\mathrm{Kur}]$ that a subset $T$ of the unit interval is a $\lambda$-set provided all countable subsets of $T$ are relatively $G_{\delta}$ in $T$.)

Lemma 4.2. It is consistent with ZFC that, simultaneously,

(i) there exists a $\lambda$-set $T \subseteq I$ of cardinality $\aleph_{2}$, and

(ii) there exists a non- $\sigma$-discrete set $E \subseteq B\left(\aleph_{2}\right)$, with $|E|=\aleph_{2}$, such that all subsets of $E$ of cardinality $\aleph_{1}$ are $\sigma$-discrete.

Let us indicate some references concerning this lemma: Jensen [Je], assuming $V=L$, shows $E\left(\omega_{2}\right)$ holds. That is, in this model $M$, there is an $\omega_{2}$ stationary set $\Lambda \subseteq \omega_{2}$ of ordinals with countable cofinality, such that all intersections $\Lambda \cap \alpha$, $\alpha<\omega_{2}$, of cofinality $\omega_{1}$, are non-stationary. Now, $E\left(\omega_{2}\right)$ is preserved under ccc extensions [F2] and there is a ccc extension to a model $M[G]$ where Martin's Axiom MA and $\aleph_{2}<2^{\aleph_{0}}$ both hold ([Ku] $)$. In this model, if $T \subseteq I$ with $|T|=\omega_{2}$, then $T$ is actually a $Q$-set (all subsets are relatively $G_{\delta}$ ) so $T$ is certainly a $\lambda$-set. 
To get (ii) in $M[G]$, let us fix, for each $\alpha \in \Lambda$, a function $x_{\alpha}: \mathbb{N} \rightarrow \omega_{2}$ with $x_{\alpha}(n) \nearrow \alpha$. Then $E=\left\{x_{\alpha}: \alpha \in \Lambda\right\} \subseteq B(\alpha)$ has the required properties (cf. [F1]; [P], Lemma p. 141).

Proposition 4.3. The statements (i) and (ii) in Lemma 4.2 yield disjoint sets $A, C$ in $I$ with $M(A, C) \times B\left(\aleph_{1}\right)$ normal but $M(A, C) \times B\left(\aleph_{2}\right)$ not normal.

Proof. We shall modify the construction from Section 2, adopting the notation, but replacing $\aleph_{1}$ by $\aleph_{2}$ and taking the sets $T$ and $E$ from (i) and (ii) of Lemma 4.2, respectively.

The arguments in Section 2 can be modified appropriately to show that $M(A, C)$ $\times B\left(\aleph_{0}\right)$ is Lindelöf and $M(A, C) \times B\left(\aleph_{2}\right)$ is not normal. To check that $M(A, C) \times$ $B\left(\aleph_{1}\right)$ is normal we shall first verify the following.

Claim. Let $H \subseteq C$ be a set of cardinality $\aleph_{1}$. Then there is a decomposition $H=\bigcup_{n=1}^{\infty} H_{n}$ and disjoint collections $\mathcal{G}_{n}$, for $n \in \mathbb{N}$, of subsets of $I \backslash A$ such that each $G \in \mathcal{G}_{n}$ intersects $H_{n}$ in a singleton and the union of every countable subfamily of $\mathcal{G}_{n}$ is a $G_{\delta}$-set in $I \backslash A$.

To this end, taking into account (1) and (2) in Section 2, let us write $H=f(L)$ with $L \subseteq E$ of cardinality $\aleph_{1}$. The set $L$ is $\sigma$-discrete in $B\left(\aleph_{2}\right)=D^{\mathbb{N}}, D$ being the discrete space of cardinality $\aleph_{2}$. Therefore, $L=\bigcup_{n=1}^{\infty} L_{n}$ with each $L_{n}$ projecting injectively into a finite product $D^{s(n)}$. By $(1), H_{n}=f\left(L_{n}\right)$ projects injectively onto a subset $Z_{n}$ of $T^{s(n)}$. Let

$$
\mathcal{G}_{n}=\left\{\{t\} \times T \times T \times T \times \cdots: t \in Z_{n}\right\} .
$$

Then each element of $\mathcal{G}_{n}$ intersects $H_{n}$ in a singleton. Since $T$ is a $\lambda$-set, so is $T^{s(n)}$ and hence for each countable $W \subseteq Z_{n}, W \times T \times T \times \cdots$ is a $G_{\delta}$-set in $T^{\mathbb{N}}=I \backslash A$ (cf. (2), Section 2). Therefore, countable unions of elements of $\mathcal{G}_{n}$ are $G_{\delta^{-}}$-sets in $I \backslash A$.

Having established the claim, let us now consider a set $F \subseteq C \times B\left(\aleph_{1}\right)$, closed in $M(A, C) \times B\left(\aleph_{1}\right)$. By Remark 2.1, it is enough to separate $F$ from $A \times B\left(\aleph_{1}\right)$ by open sets in $M(A, C) \times B\left(\aleph_{1}\right)$. Let $p: M(A, C) \times B\left(\aleph_{1}\right) \rightarrow M(A, C)$ be the projection. For any closed separable subset $S$ of $B\left(\aleph_{1}\right)$, the product $M(A, C) \times S$ is Lindelöf and hence $p(F \cap(M(A, C) \times S))$ is a countable subset of $C$. It follows that

$$
H=p(F) \subseteq C \text { has cardinality } \leq \aleph_{1} .
$$

Let $H_{n}$ and $\mathcal{G}_{n}$ be the sets and the families described in the claim. Let $Y$ be the closure of $F$ in the product $I \times B\left(\aleph_{1}\right)$. Then

$$
p(Y) \subseteq I \backslash A .
$$

Let $\mathcal{E}_{n}=\left\{p^{-1}(G) \cap Y: G \in \mathcal{G}_{n}\right\}$. Then each countable union of elements of $\mathcal{E}_{n}$ is of the form $p^{-1}(\bigcup \mathcal{A}) \cap Y$ with a countable $\mathcal{A} \subseteq \mathcal{G}_{n}$, and since $\bigcup \mathcal{A}$ is a $G_{\delta}$-set in $I \backslash A$, (2) implies that $p^{-1}(\bigcup \mathcal{A}) \cap Y$ is a $G_{\delta}$-set in $Y$; hence it is an absolute $G_{\delta}$-set.

From Lemma 4.1 we infer that each $\mathcal{E}_{n}$ is exhaustive in the space $X_{n}=p^{-1}\left(\bigcup \mathcal{G}_{n}\right)$ $\cap Y$, with the subspace topology inherited from $I \times B\left(\aleph_{1}\right)$. Since for each $c \in H_{n}$, $F \cap p^{-1}(c) \subseteq p^{-1}(G)$, where $G$ is the unique element of $\mathcal{G}_{n}$ containing $c$, it follows that the collection of fibers $\left\{F \cap p^{-1}(c): c \in C\right\}$ is $\sigma$-discretely decomposable in $I \times B\left(\aleph_{1}\right)$. 
In effect, a separation of $F$ from $A \times B\left(\aleph_{1}\right)$ in $M(A, C) \times B\left(\aleph_{1}\right)$ can be defined in the same way as in the second part of the proof of Theorem 3.3.

Now, in the opposite direction, we will show that Fleissner's axiom $\left(S C \omega_{2}\right)$ [F1, combined with $\mathrm{CH}$, guarantees the implication in the title. We refer the reader to Fremlin [Fr2] for a discussion of topics related to Fleissner's axiom.

Proposition 4.4. Assuming Fleissner's axiom $\left(S C \omega_{2}\right)$ and $C H$, if $A, C$ are disjoint sets in $I$ and $M(A, C) \times B\left(\aleph_{1}\right)$ is normal, then $M(A, C) \times S$ is normal for every completely metrizable space $S$.

Proof. As was already mentioned in the Introduction the normality of $M(A, C) \times$ $B\left(\aleph_{1}\right)$ implies that

(3) $M(A, C) \times Y$ is normal for completely metrizable spaces of weight $\leq \aleph_{1}$.

Let $S$ be an arbitrary completely metrizable space, and let $F \subseteq C \times S$ be closed in $M(A, C) \times S$. We have to separate $F$ from $A \times S$ by open sets in the product $M(A, C) \times S$ (cf. Remark 2.1).

Since we assume $\mathrm{CH}, I \backslash A$ is a union of compact sets $K_{\xi}, \xi<\omega_{1}$, such that each compact set in $I \backslash A$ is contained in some union $\bigcup_{\alpha \leq \xi} K_{\alpha}$. Let

$$
Z_{\xi}=K_{\xi} \backslash \bigcup_{\alpha<\xi} K_{\alpha}, \quad \xi<\omega_{1} .
$$

Then

$$
\text { the closure of } Z_{\xi} \text { in } I \text { is disjoint from } A \text {, and }
$$

each set in $I$ intersecting uncountably many $Z_{\xi}$ has an accumulation point in $A$.

Let $p_{1}: I \times S \rightarrow I$ be the projection onto the first coordinate. We will show that

$$
\mathcal{E}=\left\{F \cap p_{1}^{-1}\left(Z_{\xi}\right): \xi<\omega_{1}\right\} \text { is } \sigma \text {-discretely decomposable in } I \times S,
$$

where $I$ is considered with the Euclidean topology. Now, Fleissner's axiom reduces (6) to a verification of the following claim (cf. [F1], Diagram 2 on page 315). By a selector $T$ for $\mathcal{E}$, we mean a set $T \subseteq \bigcup \mathcal{E}$ such that $|T \cap E|=1$ for every $E \in \mathcal{E}$.

$$
\text { Each selector } T \text { for } \mathcal{E} \text { is } \sigma \text {-discrete. }
$$

Let $Y$ be the closure of the projection of $T$ onto the second axis (by projection $p_{2}$ ). By (3), $X=M(A, C) \times Y$ is normal so we can find an open set $U$ in $X$ containing $F \cap X$ and having no accumulation points in $A \times Y$. For any uncountable $W \subseteq T$, the projection $p_{1}(W)$ has an accumulation point in $A$ (cf. (5) and (6)). Therefore, for any $y \in Y, I \times\{y\}$ hits at most countably many sets $p_{1}^{-1}(c) \cap U$, with $c \in p_{1}(T)$. It follows that the vertical sections $U(c)=\{y \in Y:(c, y) \in U\}$ form an indexed point-countable collection $\left\{U(c): c \in p_{1}(T)\right\}$. Since each $U(c)$ is open in $Y$ an argument similar to that following (6) in Section 2 shows that $p_{2}(T)$ is $\sigma$-discrete. From this it follows that (7) is true. With (6) verified, we can now proceed again as in the second part of the proof of Theorem 3.3 to define a pair of open sets in $M(A, C) \times S$ separating $F$ from $A \times S$. That concludes the proof that $M(A, C) \times S$ is normal. 


\section{REFERENCES}

[E] R. Engelking, General Topology, Heldermann Verlag, Berlin, 1989. MR 91c:54001

[F1] W. Fleissner, An axiom for nonseparable Borel theory, Trans. Amer. Math. Soc. 251 (1979), 309-328. MR 83c:03043

[F2] W. Fleissner, Separation properties in Moore spaces, Fund. Math. 98 (1978), 279-286. MR 57:17600

[Fr1] D. H. Fremlin, Consequences of Martin's Axiom, Cambridge University Press, Cambridge, 1984. MR 86i:03001

[Fr2] D. H. Fremlin, Measure-additive coverings and measurable selectors, Dissertationes Math. 260 (1987). MR 89e:28012

[H] W. Hurewicz, Relativ Perfekte Teile von Punktmengen ung Mengen (A), Fund. Math. 12 (1928), 78-109.

[J] T. Jech, Set Theory, Academic Press, New York, 1978. MR 80a:03062

[Je] R. B. Jensen, The fine structure of the constructible hierarchy, Ann. Math. Logic 4 (1972), 229-308. MR 46:8834

[K] A. S. Kechris, Classical Descriptive Set Theory, Springer-Verlag, New York, 1995. MR 96e:03057

[Ko] G. Koumoullis, Cantor sets in Prohorov spaces, Fund. Math. 124 (1984), 155-161. MR 86c:54038

[Ku] K. Kunen, Set Theory, North-Holland, Amsterdam, 1980. MR 82f:03001

[Kur] K. Kuratowski, Topology, Volume 1, Polish Scientific Publishers, Warsaw, 1966. MR 36:840

[L1] L. B. Lawrence, The influence of a small cardinal on the product of a Lindelöf space with the irrationals, Proc. Amer. Math. Soc. 110 (1990), 535-542. MR 90m:54014

[L2] L. B. Lawrence, A ZFC example (of minimum weight) of a Lindelöf space and a completely metrizable space with a nonnormal product, Proc. Amer. Math. Soc. 124 (1996), 627-632. MR 96d:54005

[M1] E. A. Michael, The product of a normal space and a metric space need not be normal, Bull. Amer. Math. Soc. 69 (1963), 375-376. MR 27:2956

[M2] E. A. Michael, Paracompactness and the Lindelöf property in finite and countable cartesian products, Compositio Math. 23 (1971), 199-214. MR 44:4706

[M3] E. A. Michael, A note on completely metrizable spaces, Proc. Amer. Math. Soc. 96 (1986), 513-522. MR 88a:54068

[P] R. Pol, Note on decompositions of metrizable spaces II, Fund. Math. 100 (1978), 129-143. MR 58:12953

[RS] M. E. Rudin and M. Starbird, Products with a metric factor, Gen. Top. Appl. 5 (1975), 235-248. MR 52:1606

[S] A. Stone, On $\sigma$-discreteness and Borel isomorphisms, Amer. J. Math. 85 (1963), 655-666. MR 28:33

Department of Mathematics and Statistics, Miami University, Oxford, Ohio 45056

E-mail address: dburke@miavx1.muohio.edu

Department of Mathematics, Warsaw University, Warsaw, Poland

E-mail address: pol@mimuw.edu.pl 